\section{PSQ-222 ANTICHOLINERGIC RISK IN THE ELDERLY}

AB Morillo Mora*, V Gonzalez Rosa. Hospital Serranía De Málaga, Pharmacy, Malaga, Spain

\subsection{6/ejhpharm-2021-eahpconf.341}

Background and importance Anticholinergic drugs (ACD) are closely related to serious negative health outcomes in the elderly but they are widely used in these patients. There are several scales in the literature to predict the risk of suffering anticholinergic effects in the elderly.

Aim and objectives To analyse the anticholinergic risk and its variability using different scales in a sample of institutionalised elderly patients.

Material and methods An observational retrospective study was conducted in institutionalised patients with the following characteristics: age $>65$ years, polymedicated ( $>5$ drugs) with at least one prescribed ACD. The variables collected were: age, sex, prescribed drugs and anticholinergic risk calculated from the anticholinergic cognitive burden scale (CBA), anticholinergic risk scale (ARS), Chew's scale (Chew), anticholinergic drug scale (ADS), Duran's scale (Duran) and drug burden index (DBI). Data were obtained from the electronic clinical history. Results 41 patients $(73.2 \%$ women $)$ were included with a mean age of $86.6 \pm 7.1$ years. Mean prescriptions were 11.2 \pm 2.7 . Percentage of patients with at least one ACD prescribed according to the different scales was: $75.6 \%$ (CBA), $61 \%$ (ARS), 56.1\% (Chew), 73.2\% (ADS), 70.7\% (Duran), 90.2\% (DBI). Percentage of patients with low anticholinergic risk was: $31.7 \%$ (CBA), 34.1\% (ARS), 24.4\% (Chew), 31.7\% (ADS), 34.1\% (Duran), 0\% (DBI). Percentage of patients with medium anticholinergic risk was: 9.8\% (CBA), 14.6\% (ARS), 12.2\% (Chew), 26.8\% (ADS), 0\% (Duran), 24.4\% (DBI). Percentage of patients with high anticholinergic risk was: $34.1 \%$ (CBA), $12.2 \%$ (ARS), $19.5 \%$ (Chew), $14.6 \%$ (ADS), 36.6\% (Duran), 65.9\% (DBI).

Conclusion and relevance There was a high probability of anticholinergic effects in our sample of patients but according to the scale used, both the percentage of patients at risk of anticholinergic effects and the degree of the risk were variable. It seems that the DBI scale tended to detect greater risk in our patients. Further studies are needed to validate which scale is the most appropriate for our population.

\section{REFERENCES AND/OR ACKNOWLEDGEMENTS}

Conflict of interest No conflict of interest

\section{PSQ-223 DRUGS TO AVOID. AN OPPORTUNITY IN HEALTHCARE PATIENTS: CHECKING PRESCRIRE'S RECOMMENDATIONS}

J Fernández-Bravo Rodrigo*, A Flor Garcia, L Martínez Valdivieso, S Canales Ugarte, G Marcos Pérez, D Barreda Hernandez. Hospital Virgen De La Luz, Pharmacy, Cuenca, Spain

\subsection{6/ejhpharm-2021-eahpconf.342}

Background and importance There are drugs that should be avoided despite authorisation by agencies. Can we justify drugs with no proven efficacy or relevant clinical outcomes? Prescrire (non-profit organisation, IF: 0.18) publishes an annual review of drugs to avoid. They identified 105 drugs that cover studies between 2010 and 2019. Drugs with a better harm-benefit balance are available in most of cases.
Aim and objectives To analyse and check our pharmacotherapeutic guide (PG) according to the 2020 Prescrire's review.

Material and methods We evaluated the 2020 annual review of drugs to avoid from Prescrire's and its concordance with a PG of a level II hospital and the impact of use and cost versus alternatives available, between January and August 2020. Information was collected from Farmatools software and the Prescrire's review. Data recollected were: drugs and Prescrire's alternative proposal included in the PG; use (defined daily dose (DDD)) and cost of the drugs of Prescrire's included in the PG and the alternatives and the entire PG.

Results 105 drugs were studied. 23 were in the PG (21.9\%), 15 with a guaranteed better choice, all of them in the PG (100\%). 2 of 23 (trabectedin, vinflunine) had any use. 20 of 23 had 8592 DDD, 5421.8€ versus 1507871 DDD, 110 $327 €$ in $13 / 15$ alternatives. 1 of 23 (teriflunomide) had 4580 DDD, $132179 €$ versus 1907 DDD, $184296 €$ for the alternative (IFN- $\beta$ ). Global PG use was around $14000000 € .82$ drugs were not in the PG (78.1\%). 60 of them had better harm-benefit alternative; 58 (96.7\%) were in our PG.

Conclusion and relevance Our study showed that our PG was well adapted according to Prescrire's recommendations. The presence of drugs to avoid was low, the majority because their use was well established in clinical practice or are reserved for very specific situations. The global impact (without teriflunomide) in total cost and use was worthless. Our next step is to re-evaluate the results and make a better PG, with a special focus on teriflunomide (currently has a strong follow-up) and minimise the use of those essential to the physician in very specific situations. It is possible to conclude that our PG, developed by hospital pharmacists in a multidisciplinary team, guarantees quality, safety and efficiency.

\section{REFERENCES AND/OR ACKNOWLEDGEMENTS}

1. Towards better patient care: drugs to avoid in 2020. Prescrire Int 2020;29 (212):51-1-51-10.

Conflict of interest No conflict of interest

\section{PSQ-224 CLINICAL EXPERIENCE OF PEMBROLIZUMAB WITH AXITINIB IN RENAL CELL CARCINOMA}

S Rotea*, T Calleja, F Busto, M Mateos, E Fernandez, I Martin. Hospital Pharmacy, Complejo Hospitalario Universitario A Coruña, A Coruña, Spain

\subsection{6/ejhpharm-2021-eahpconf.343}

Background and importance There are several types of renal cell tumours, the most frequent being clear cell renal carcinoma (ccRCC) which represents $80 \%$ of malignant renal tumours in adults. Pembrolizumab, in combination with axitinib, is indicated for the firstline treatment of advanced RCC in adults.

Aim and objectives To describe and analyse the effectiveness and safety of pembrolizumab and axitinib in a tertiary hospital clinical practice.

Material and methods An observational retrospective study was conducted in all patients diagnosed with ccRCC and treated with pembrolizumab and axitinib from March 2019 to October 2020. All patients gave their informed consent. Data sources were the electronic medical records. Variables analysed were: sex, age, PDL-1, prior lines of treatments, IMDC risk and presence of metastases at the start therapy, duration of treatment and interruption causes, grade and type of toxicities and best TAC response. 
Results 13 patients were included, 76.9\% men, mean age 60.4 \pm 8.8 years. Among the four patients with tumour samples that were evaluated for PD-L1 expression, $75 \%$ had a score $\geq 1$. Three patients were treated with at least one previous line. Previous treatments were: sunitinib $(n=3)$ cabozantinib $(n=1)$ or nivolumab $(\mathrm{n}=1)$. IMDC risk classification: $7.7 \%$ favourable, $53.8 \%$ intermediate and $38.5 \%$ poor risk. Presence of metastases: lung (7/13), bone (5/13), liver (3/13), ganglionar (2/13), cerebral (1/13) and unknown (2/13). All patients were treated with pembrolizumab $200 \mathrm{mg}$ every 3 weeks and axitinib $5 \mathrm{mg}$ twice daily until progression, unacceptable toxicity or death. Mean duration of treatment was 28.7 weeks. $46 \%$ are continuing with active treatment. Discontinuation causes included: death $(n=3)$, adverse effects $(n=3)$ and progression $(n=1)$. Toxicities included: asthenia grade $(\mathrm{G}) 1-3 \quad(\mathrm{n}=11)$, anorexia G1-2 $(n=6)$, diarrhoea G1-4 $(n=5)$, liver profile alterations G1-3 $(\mathrm{n}=3)$, hyperthyroidism G1-3 $(\mathrm{n}=3)$, abdominal pain G1-2 $(n=3)$, palmar-plantar erythrodysesthesia G2-3 $(n=2)$, pruritus G1 $(n=1)$, dizziness and paraesthesia G1 $(n=1)$, vomiting G1 $(n=1)$, thrombopenia G2 $(n=1)$ and arthralgias G1 $(n=1)$. Best TAC responses obtained were: 50\% stable disease, 25\% partial response and $12.5 \%$ progressive disease. In five patients the response rate was not evaluated.

Conclusion and relevance Effectiveness in our patients resulted in a higher objective response rate than that in the KEYNOTE-426 trial. The combination treatment was well tolerated. To rationalise the use of novel medicines and optimise efficiency, measuring health results is crucial.

\section{REFERENCES AND/OR ACKNOWLEDGEMENTS}

Conflict of interest No conflict of interest

\section{PSQ-225 ELDER FALLERS: A GROUP AT RISK OF READMISSION?}

${ }^{1} \mathrm{C}$ Ratsimbazafy*, ${ }^{1} \mathrm{C}$ Schwab, ${ }^{2} \mathrm{~N}$ Richebourg, ${ }^{2} \mathrm{O}$ Descours, ${ }^{2} \mathrm{Q}$ Law-Koune, ${ }^{1} \mathrm{C}$ Fernandez, ${ }^{1} \mathrm{P}$ Hindlet. ${ }^{1} A p h p$.Sorbonne Université-Inserm-Institut Pierre Louis D'épidémiologie Et De Santé Publique, Pharmacy, Paris, France; ${ }^{2}$ Aphp Sorbonne Universite, Pharmacy, Paris, France

\subsection{6/ejhpharm-2021-eahpconf.344}

Background and importance Readmission of elderly patients is an issue of concern for both healthcare professionals and health authorities. The observed rate of unscheduled 30 day readmission is up to $14 \%$ in patients aged 75 years or over. Moreover, the proportion of readmissions deemed avoidable is estimated at $23 \%$. In elderly patients, falls are frequent and can lead to consultations in the emergency department (ED) or even hospitalisation. The proportion of people hospitalised after visiting the ED for a fall increases with age: from $25 \%$ at 65 years to almost half at 90 years. At the end of the index hospital stay, readmission of older fallers are thus challenging for the healthcare system.

Aim and objectives To describe older patients hospitalised for falls and identify the risk of readmission in that population.

Material and methods We conducted an observational, single centre, prospective study (from April to June 2019). Inclusion criteria were: patients aged 75 and over, admitted to the ED for falls and consenting to the study. For patients subsequently hospitalised, geriatric scores were determined (risk of readmission (ISAR score), state of frailty, degree of autonomy (Katz score)), and when appropriate, medication treatments were listed and compliance of patients was assessed (Girerd score).

Results During this 3 month study, 154 patients were included (median age 86 years (min $75-\max 103$ ), sex ratio 0.44 ), of whom 73 patients were hospitalised. Among these patients, $63 \%$ presented a high risk of readmission; $45.2 \%$ were at risk of frailty; $72.6 \%$ were dependent. Finally, 53 of the 73 patients $(72.6 \%)$ had medications in the primary care setting and presented a $71.7 \%$ non-compliance or low compliance rate. 58 patients $(79.5 \%)$ had at least one drug that can cause falls (min 1-max 7).

Conclusion and relevance Older patients presenting at hospital with a fall were often likely to become frail and the majority were dependent. More importantly, this population was at high risk of readmission. Therefore, future studies are now needed to test interventions aimed at reducing this risk.

\section{REFERENCES AND/OR ACKNOWLEDGEMENTS}

Conflict of interest No conflict of interest

\section{PSQ-226 ANALYSIS OF HIGH ALERT MEDICATION PRESCRIPTIONS IN A NURSING HOME}

${ }^{1} \mathrm{R}$ Sánchez Del Moral, 'I García Giménez, ${ }^{1}$ A Peláez Bejarano*, ${ }^{2} E M$ Talero Barrientos. ${ }^{1}$ Hospital Juan Ramón Jiménez, Pharmacy Department, Huelva, Spain; ${ }^{2}$ University of Seville, Pharmacy Faculty-Pharmacology Department, Seville, Spain

\subsection{6/ejhpharm-2021-eahpconf.345}

Background and importance High alert medications are those that, when they are not being properly used, are more likely to cause serious or even fatal harm to patients. Chronic patients are especially vulnerable to these possible errors because of their comorbidity and polypharmacy. The Ministry of Health, Social Services and Equality of Spain promotes the implementation of improving safe practices for those patients. In 2014, a panel of experts developed a list of high alert drugs for chronic patients to prioritise practices for improving safety in these patients. This list was named the HAMC list (high alert medications for patients with chronic illnesses) and was published by the Ministry of Health, Social Services and Equality of Spain.

Aim and objectives To analyse the prevalence of prescribed medications included in the HAMC list in a nursing home.

Material and methods A descriptive, transversal, retrospective study was carried out in September 2020 that included all residents with chronic illnesses in a nursing home assigned to our pharmacy service. Variables recorded were: demographic data, number of prescribed medicaments, and number and type of prescribed medicaments included in the HAMC list. Results 81 patients were included (59 men) with a mean age of 72 (56-94) years. 721 drugs were prescribed, and 186 were included in the HAMC list. At least 1 HAMC was prescribed in $86 \%$ of patients, with a mean of 3 HAMC per patient (1-7). The therapeutic groups of the drugs on the HAMC that were prescribed were: benzodiazepines $(63 \%$ of patients), inhibitors of platelet aggregation (36\%), antipsychotics (26\%), beta-adrenergic blockers (26\%), oral hypoglycaemics agents (26\%), loop diuretics (19\%), oral anticoagulants (11\%), antiepileptics (9\%), opioids (9\%), including minor and major opioids, insulin (7\%), eplerenone/spironolactone (7\%), immunosuppressants (1\%) and non-steroidal anti-inflammatory drugs (1\%). 